\title{
Energy-efficient control of solid-state lighting
}

\section{Joseph A. Paradiso, Matthew Aldrich, and Nan Zhao}

Aided by sensors, machine-optimized control of intensity and color yields additional energy savings.

Lighting control is in the midst of radical change. Presentday state-of-the-art lighting systems tend to be extremely complex. They exhibit very flexible actuation possibilities with many degrees of freedom that can be exploited to answer dynamiclighting needs. However, user interfaces to these systems are woefully lacking. They are frequently denigrated to a panel of buttons that select particular presets that are often cryptically defined, poorly labeled, and seldom desirable. Higher-end lighting systems can provide affordances such as a touch screen to select particular, graphically illustrated presets, but the straitjacketed assumptions that are made often lead to frustration. Most attempts to integrate sensor feedback into commercial systems exploit simple motion sensors that tend to activate all lighting in a space when one occupant moves. This is generally energy wasteful. The converse, turning off all lights when the occupant does not move for a while, may also be a wrong choice that can irritate the user.

Today's crude motion sensors represent a coarse attempt at leveraging limited information and simple context to reduce energy consumption. Balancing precise control and energy efficiency remains a goal in modern lighting systems, ${ }^{1-10}$ since lighting accounts for $22 \%$ of all electricity consumed in the United States. ${ }^{11}$

The inherent control flexibility implied by solid-state lighting-combined with a rich description of a user's environment provided by emerging sensor networks-offers a chance to rethink our present modes of lighting control. It also requires us to consider the importance of color and efficacy. ${ }^{12-18}$ Building upon the inherently digital nature of this technology, we discuss our vision of lighting control and suggest several highly responsive schemes that are adept at meeting users' needs while mitigating energy usage.

Our research aims at minimizing the energy spent lighting while simultaneously maximizing the light source's usefulness.

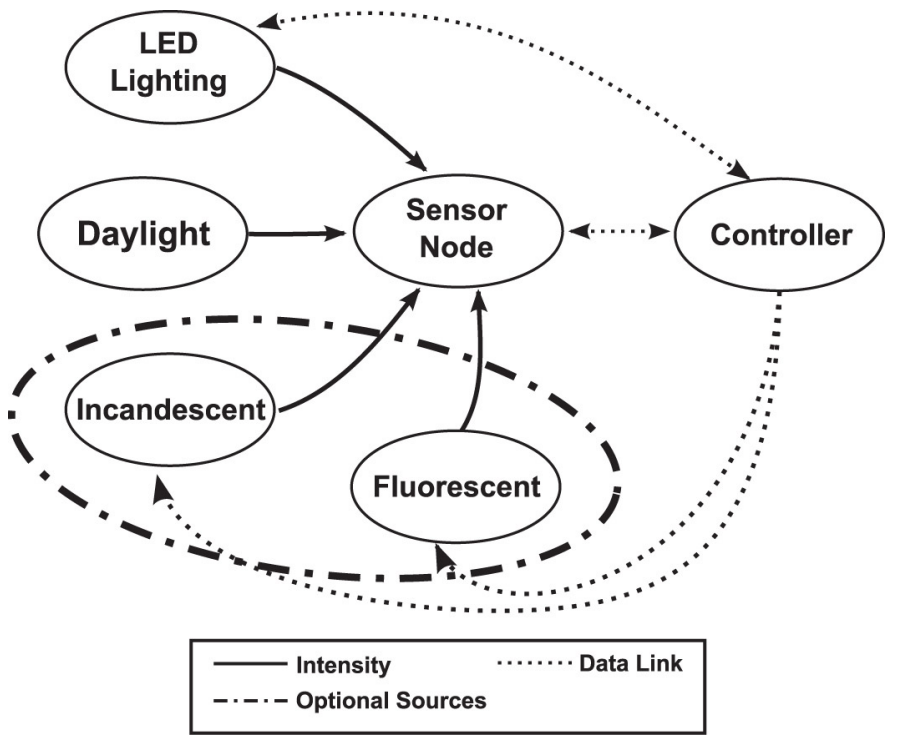

Figure 1. The lighting network consists of LED light sources, optional incandescent and fluorescent sources, and ambient room conditions (daylight), that are measured by a single or a group of sensor nodes. The latter return intensity and color information to the control node (e.g., a computer) for processing. Here, the intensity is controlled by a link to the artificial-light sources that is bidirectional from either the sensor node or the LEDs.

We recently provided two examples of strategies for intensity and color control of solid-state lighting networks. ${ }^{19}$ Our system computes the optimal intensity and color by incorporating illuminance feedback from a portable, photosensitive device (see Figure 1). Rather than force the user to adjust each light source manually, the user-aided by the photosensitive node-takes an illuminance snapshot of their work surface. We use this to infer the contributions of both the adjustable (independently for each modulatable fixture) and nonadjustable lighting (i.e., daylight). In turn, the control scheme calculates the energy-optimal intensities for the overhead lighting 


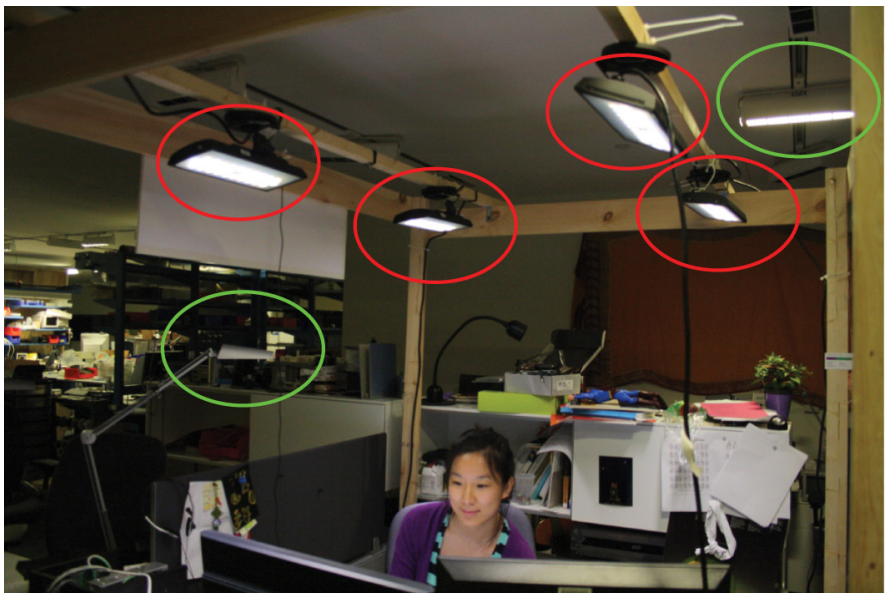

Figure 2. We constructed a testbed of adjustable LED sources (red) and other uncontrolled sources (green). If the photosensor measures differences in ambient lighting, the control system adjusts the overhead lighting in real time. For example, if one of the sources circled in green were turned on or off, or if daylight levels changed, the closed-loop control would minimize this difference. This control is complementary to the initial intensity set by the linear program.

network. These contributions represent the 'importance' of the light source. This data, along with models of power consumption and the rated fixture intensity, help guide the optimization routine. For our phosphor-converted testbed, a simple, linear program efficiently calculates the intensity of each luminaire (see Figure 2).

We minimize the total power consumed by the network, so that our cost vector is the measured current of each source. The activity vector is the stored reference intensity weighted by the most recent specific contribution measurements. The desired illuminance (less ambient light) is used to constrain the linear program, i.e., to minimize the forward current without violating the user's illuminance. The linear program favors adjusting the fixtures that offer the greatest contribution, as measured by the photosensor.

Of course, even after the lighting is optimized, the user is free to make changes. In this case, the updated illuminance constraint is solved again using the previous activity vector until another room measurement is taken. Using these techniques, we conducted two short studies to test the algorithm's ability to track the user-specified illuminance over an eight-hour period (see Figure 3). The average energy savings were 90 and $60 \%$, respectively.

We also presented a method of color control for hybrid, active-emitter and phosphor-converted lighting systems that exceeds the photometric results typical of multiwavelength sys- tems solved using exact and minimum-square error solutions. We developed light sources that include typical red, green, and blue LEDs, but added phosphor-converted amber and cyan LEDs to create a five-wavelength source. ${ }^{19}$ Mathematically, solutions of these overdetermined systems are solved by minimizing some error criteria (most notably, using the pseudoinverse). We selected a gradient-free technique, mesh adaptive search, ${ }^{20}$ to calculate the five LED setpoints for a given intensity and color temperature for each light source.

Most notably, by decreasing or increasing the distance from the blackbody curve $(\Delta u v)$, this technique allowed us to generate solutions that maximized either the light source's colorrendering index or its efficacy. In broad terms, the algorithm is capable of efficiently finding solutions of the same color temperature and intensity, yet comprised of different wavelengths. In empirical testing on the actual prototypes, we achieved an average color-rendering index greater than 90 (out of 100) for 11 whitepoints at three unique intensities. We also measured a $28 \%$ improvement in efficacy by expanding the $\Delta u v$ parameter.

Of course, these improvements come at a cost: typical whitepoints (depending on the color space) require $\Delta u v \leq .05$, which will impact the savings in practice. Figure 4 compares empirical data based on our technique and others. Because of the complexity of the search, this method is not suitable for run-time control. In our case, we stored the results locally in flash memory on the microcontroller. While these results are promising, more investigation is required. Our dimming strategy using personalized controls simplifies adjustment of a network of fixtures, but still requires some user interaction and accounts for lighting at specific points measured by discrete sensors. Better strategies will focus on the required technology and computation to limit these manual processes while accounting for lighting sampled over larger areas through use of other sensor systems (e.g., wearable sensors or embedded cameras). Accounting for multiple users presents additional challenges in large areas. In addition, energy savings may be attributable to other factors, such as how often the user performs an adjustment and the proximity of the testbed to office windows.

And while systems that can seamlessly adjust between modes of high color rendering and efficacy are intriguing, the possible energy savings relative to advanced dimming control do not warrant the system's complexity. However, as an improvement over existing systems, our five-wavelength source guarantees exceptional color rendering over a very broad range of color temperature.

Continued on next page 

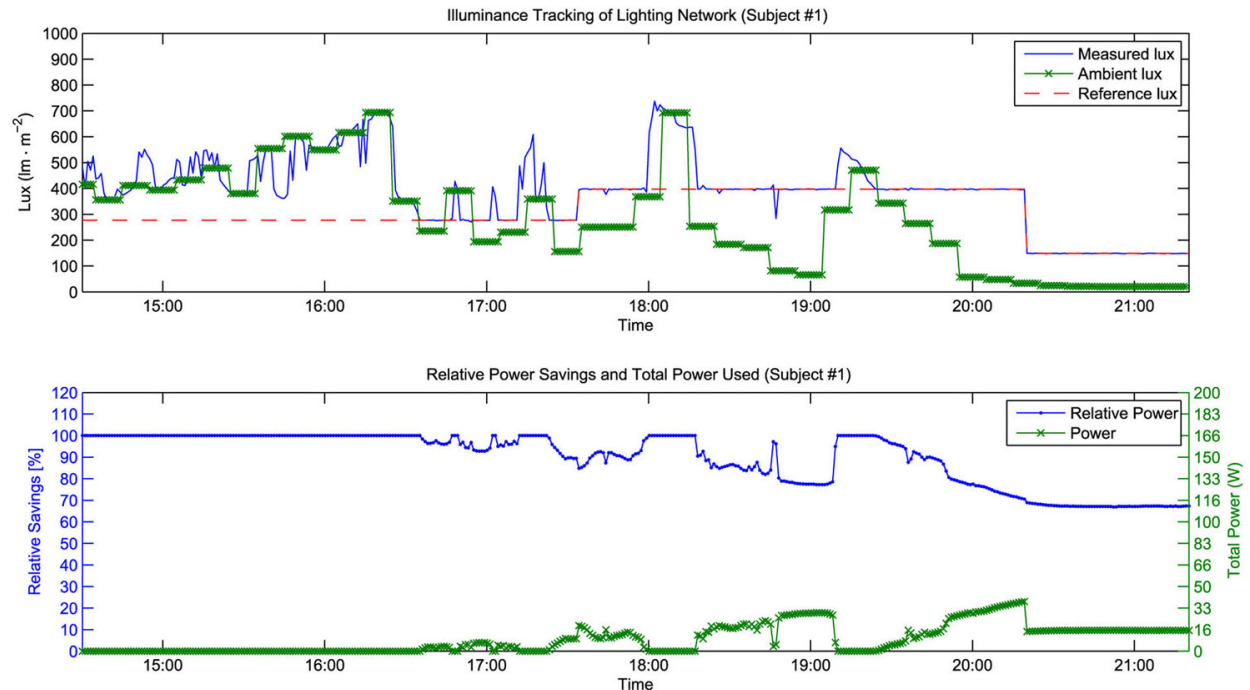

(a)
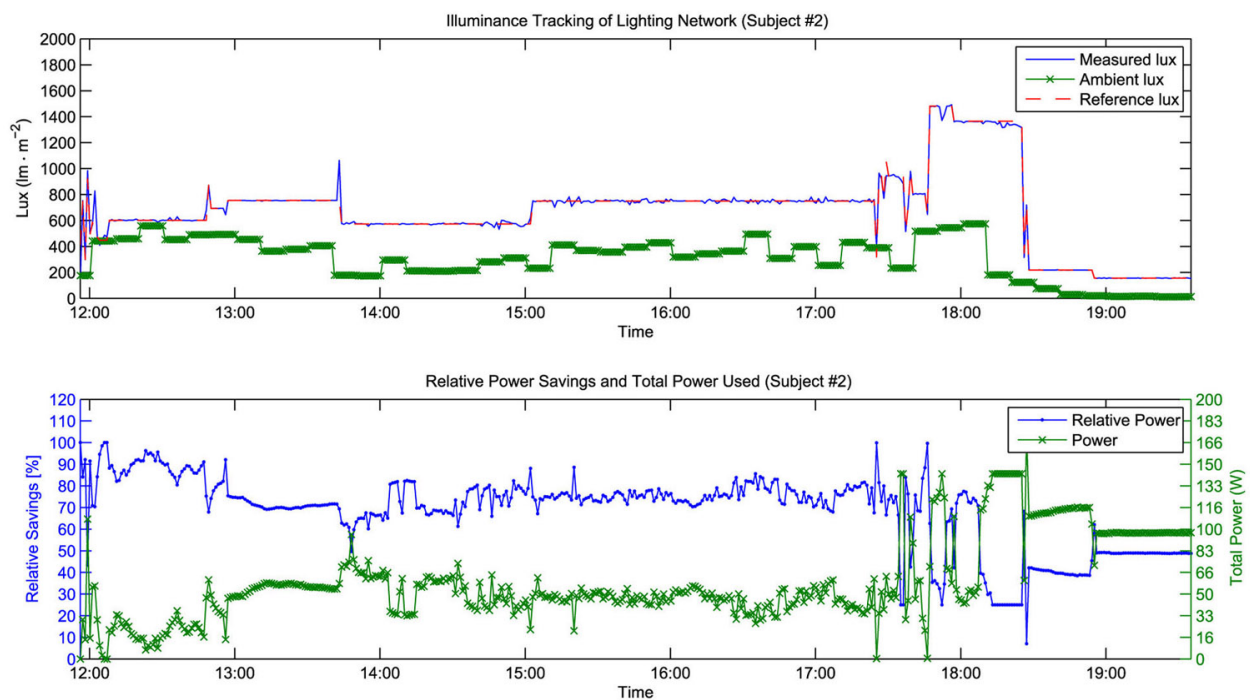

(b)

Figure 3. Test results for two subjects using our phosphor-based lighting network. The relative power savings are based on the lighting network set to maximum intensity (analogous to fluorescent lighting in an office). The reference lux is set by adjusting the illuminance slider on the sensor board. In (b), at approximately 17:30, subject \#2 made several rapid changes to the reference illuminance. We see that the system tracks these changes well. Ambient light was logged every 10 minutes, while sensor data was logged every minute. The closed-loop control ran at approximately $10 \mathrm{~Hz}$.

Other activity in our research group ${ }^{21}$ focuses on similarly obsoleting the concept of the thermostat, exploiting low-power, wearable, and in situ sensing to drive heating, ventilating, and air-conditioning (HVAC) systems through models of inferred user comfort rather than temperature at one point on the wall. We aim to merge both our lighting and HVAC efforts in the near future to achieve a unified sensing, control, an intuitive user- interface framework that will seamlessly and efficiently manage building utilities to meet both inferred and explicitly defined user goals. 

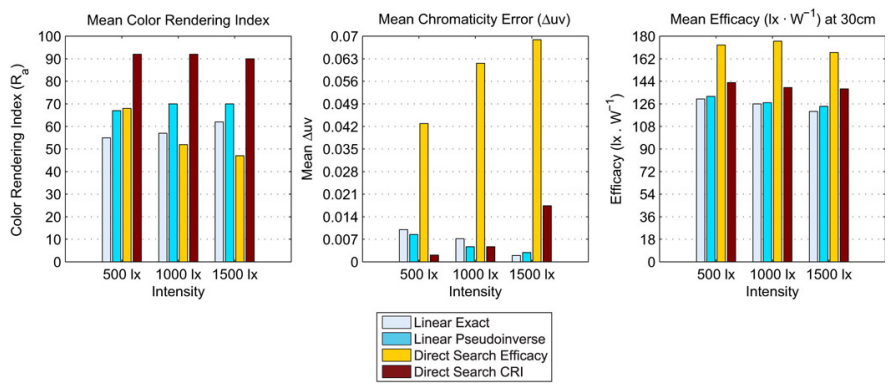

Figure 4. Results obtained from studies of linear and nonlinear methods of color control with the spectrometer head positioned $30 \mathrm{~cm}$ from the test source. Parameter averages were taken for the 11 tested white points. We see the effectiveness of the direct search algorithm, which is capable of outperforming the exact and overdetermined methods of control with respect to the color-rendering index (CRI or $\left.R_{a}\right)$. If efficacy is maximized, this technique creates white points $28 \%$ more efficiently at the cost of an increased distance from the blackbody curve $(\Delta u v)$ error. Alternatively, rather than minimizing the $\Delta u v$ error in the objective function, $\Delta u v$ could be specified as a nonlinear inequality constraint at the cost of completion time. Note the marginal improvement of the overdetermined over the exact method. The average time of completion for each white point was approximately 15-30s on an Intel Core 2 Quad, making this a feasible method for factory calibration.

We wish to thank John Warwick and the team at Philips-Color Kinetics for donation of color-adjustable white fixtures for the control testbed. We also want to thank the Responsive Environments Group at the MIT Media Laboratory for their insight and help during testing. This research is directly funded by the MIT Media Laboratory.

\section{Author Information}

\section{Joseph A. Paradiso}

Massachusetts Institute of Technology (MIT)

Cambridge, MA

Joseph Paradiso is an associate professor of media arts and sciences at the MIT Media Laboratory and director of the Responsive Environments group, which explores how sensor networks augment and mediate human experience, interaction, and perception. He has a PhD in physics from the MIT Laboratory for Nuclear Science.

\section{Matthew Aldrich and Nan Zhao}

MIT Media Laboratory

MIT

Cambridge, MA

\section{References}

1. V. H. C. Crisp, Preliminary study of automatic daylight control of artificial lighting, Light. Res. Technol. 9 (1), pp. 31-41, 1977. doi:10.1177/096032717700900104 2. V. H. C. Crisp, The light switch in buildings, Light. Res. Technol. 10 (2), pp. 69-82, 1978. doi:10.1177/096032717801000202

3. V. H. C. Crisp and G. Henderson, The energy management of artificial lighting use, Light. Res. Technol. 14 (4), pp. 193-206, 1982. doi:10.1177/096032718201400402

4. D. R. G. Hunt, Simple expressions for predicting energy savings from photoelectric control of lighting, Light. Res. Technol. 9 (2), pp. 93-102, 1977 doi: $10.1177 / 096032717700900204$

5. D. R. G. Hunt, Improved daylight data for predicting energy savings from photoelectric controls, Light. Res. Technol. 11 (1), pp. 9-23, 1979. doi:10.1177/14771535790110010301

6. M. C. Mozer, The neural network house: an environment that adapts to its inhabitants, Proc. Am. Assoc. Artif. Intell. Spring Symp. Intell. Environ., pp. 110-114, 1998.

7. H. Park, J. Burke, and M. B. Srivastava, Design and implementation of a wireless sensor network for intelligent light control, Proc. $6^{\text {th }}$ Int'l Conf. Inf. Process. Sens. Netw. (IPSN'07), pp. 370-379, 2007. doi:10.1145/1236360.1236407

8. M. Miki, A. Amamiya, and T. Hiroyasu, Distributed optimal control of lighting based on stochastic hill climbing method with variable neighborhood, ISIC/IEEE Int'1 Conf. Syst. Man Cybernet., pp. 1676-1680, 2007. doi:10.1109/ICSMC.2007.4413957

9. M.-S. Pan, L.-W. Yeh, Y.-A. Chen, Y.-H. Lin, and Y.-C. Tseng, Design and implementation of a WSN-based intelligent light control system, IEEE Int'l Conf. Distr. Comput. Syst. Wkshps, pp. 321-326, 2008. doi:10.1109/ICDCS.Workshops.2008.35

10. S. Bhardwaj, T. Ozcelebi, and J. Lukkien, Smart lighting using LED luminaries, $8^{\text {th }}$ IEEE Int'l Conf. Pervas. Comput. Commun. (PERCOM) Wkshps, pp. 654-659, 2010 .

11. Navigent Consulting Inc., Solid-state lighting research and development portfolio: multi-year program plan $F Y^{\prime} 09-F Y^{\prime} 15$ tech. rep., Lighting Research and Development Building Technologies Program, Office of Energy Efficiency and Renewable Energy, US Department of Energy, 2009.

12. A. Žukauskas, R. Vaicekauskas, F. Ivanauskas, R. Gaska, and M. S. Shur, Optimization of white polychromatic semiconductor lamps, Appl. Phys. Lett. 80 (2), pp. 234 236, 2002. doi:10.1063/1.1432107

13. N. Narendran, L. Deng, R. M. Pysar, Y. Gu, and H. Yu, Performance characteristics of high-power light-emitting diodes, Proc. SPIE 5187, pp. 267-275, 2004. doi:10.1117/12.515647

14. E. F. Schubert and J. K. Kim, Solid-state light sources getting smart, Science 308 (5726), pp. 1274-1278, 2005. doi:10.1126/science.1108712

15. R. Stanikūnas, H. Vaitkevičius, A. Švegžda, V. Viliūnas, Z. Bliznikas, K. Breive, R. Vaicekauskas, A. Novičkovas, et al., Polychromatic solid-state lamps versus tungsten radiator: hue changes of Munsell samples, J. Phys. D: Appl. Phys. 38 (17), pp. 3202 3207, 2005. doi:10.1088/0022-3727/38/17/S23

16. Z. Lei, G. Xia, L. Ting, G. Xiaoling, L. Q. Ming, and S. Guangdi, Color rendering and luminous efficacy of trichromatic and tetrachromatic LED-based white LEDs, Microelectron. J. 38 (1), pp. 1-6, 2007. doi:10.1016/j.mejo.2006.09.004

17. A. Žukauskas, R. Vaicekauskas, F. Ivanauskas, H. Vaitkevičius, and M. S. Shur, Rendering a color palette by light-emitting diodes, Appl. Phys. Lett. 93 (2), p. 021109, 2008. doi:10.1063/1.2955843

18. K. J. Dowling and B. Kolsky, The design of a spectrally tunable light source, Proc. SPIE 7422 (1), p. 742206, 2009. doi:10.1117/12.829380

19. M. Aldrich, N. Zhao, and J. Paradiso, Energy efficient control of polychromatic solid state lighting using a sensor network, Proc. SPIE 7784, p. 778408, 2010 doi: $10.1117 / 12.860755$

20. C. Audet and J. E. Dennis Jr, Mesh adaptive direct search algorithms for constrained optimization, Soc. Industr. Appl. Math. J. Optim. 17 (1), pp. 188-217, 2006 doi:10.1137/040603371

21. M. Feldmeier and J. A. Paradiso, Personalized HVAC control system, Internet of Things (IOT), pp. 1-8, 2010. doi:10.1109/IOT.2010.5678444 